\title{
Analysis of Reflector Antennas in Radio Telescopes
}

\section{Kim Ho Yeap*1, Mey Chern Loh ${ }^{1}$, Choy Yoong Tham ${ }^{2}$, Chorng Yin Yiam ${ }^{1}$, Kee Choon Yeong ${ }^{1}$ and Koon Chun Lai ${ }^{1}$}

\author{
${ }^{1}$ Faculty of Engineering and Green Technology, Tunku Abdul Rahman University, Jalan Universiti, Bandar Barat, 31900 \\ Kampar Perak, Malaysia. \\ ${ }^{2}$ School of Science and Technology, Wawasan Open University, 54 Jln. Sultan Ahmad Shah, 10050 Penang, Malaysia. \\ *corresponding author, E-mail: yeapkh@utar.edu.my
}

\begin{abstract}
We present an analysis on the performance of the Cassegrain and Gregorian on-axis, off-axis and offset antennas. In our study, we have adopted the design parameters for the Cassegrain configuration used in the Atacama Large Millimeter Array (ALMA) project. Modifications on the original parameters are made so as to meet the design requirement for the off-axis and offset configurations. To reduce spillover loss in the offset antennas, we have adjusted the angle between the axis of the primary reflector and that of the sub-reflector, so that the feed horn is placed right next to the edge of the primary reflector. This is to allow the offset antennas to receive the highest power at the feed horn. In our study, signal frequency at the high-edge of ALMA band 1, i.e. $45 \mathrm{GHz}$ has been selected. The results obtained from the physical optics simulation show that the radiation characteristics of both Cassegrain and Gregorian antennas are similar. The on-axis designs exhibit the best performance with an aperture efficiency $\varepsilon_{a}$ as high as $80.34 \%$. This is followed by the offset designs with $\varepsilon_{a}=80.24 \%$ and $80.26 \%$ for the Cassegrain and Gregorian configurations, respectively. Due to significant spillover loss at the centre hole of the primary reflector, the off-axis designs give the lowest main lobe and high side lobes. The performance of the off-axis configurations is therefore the poorest, with $\varepsilon_{a}$ below $75 \%$. Our analysis also shows that the performances of both onaxis Cassegrain and Gregorian antennas are comparable to each other.
\end{abstract}

\section{Introduction}

Radio telescopes are built to observe naturally occurring signal emission from cosmic sources, such as stars, galaxies, planet, quasars, etc $[1-5]$. Signals from distant celestial objects received at the surface of the earth are usually very faint. Hence, the size of a radio telescope has to be large so as to increase the signal energy received. A typical radio telescope consists of a parabolic primary reflector antenna and a hyperboloid or ellipsoid sub-reflector. The large circular parabolic reflector is to ensure that the telescope has a large signal collecting aperture and to give high angular resolution over a wide frequency range. Incoming signal collected by the primary reflector is focused onto a feed horn located behind or below the parabolic primary reflector. The incoming signal is then coupled onto a detector mounted in the waveguide and processed to display the spectral and spatial information [6-9].

Reflector antennas' design could have different forms of geometrical configurations. The most commonly found ones are the prime-focus, Cassegrain and Gregorian configurations [10]. The prime-focus antenna is the simplest. It consists of only a main reflector and feed horn mounted directly at the focal point, with its aperture facing downward towards the reflector. The Cassegrain and Gregorian antennas, on the other hand, are multiple-reflector antennas, i.e. they consist of at least two reflectors. Unlike the case of the prime-focus, the feed of the Cassegrain or Gregorian configuration is usually placed at the bottom, some distance below the primary reflector. Multiple-reflector antennas are usually more popular. They are preferred over the primefocus system for a number of reasons. Firstly, the magnifying sub-reflector can increase the effective focal length $f$ to reflector diameter $D$ ratio ( $f / D$ ratio), allowing an array of multiple feed horns to be located at the focal plane. Secondly, the feed horn of the Cassegrain and Gregorian configurations is directed towards the cold sky, instead of the warm ground. It therefore avoids picking up thermal noise from the ground. Also, the receivers can be easier accessed since they are located near the vertex of the primary reflector, instead of at the primary focal point. Examples of existing telescopes which employ the Gregorian configuration are the Green Bank Telescope [1114], the Arecibo observatory $[15,16]$, the Allen Telescope Array (ATA) [17, 18], and the Square Kilometer Array (SKA) interferometer currently being developed in both Australia and South Africa [19 - 22]. On the other hand, Cassegrain configurations are used in the design of the sixtysix $12 \mathrm{~m}$ antennas in the Atacama Large Millimeter Array (ALMA) project, where the most sophisticated millimeter/submillimeter interferometer is built in northern Chile [23 - 26]. The Crawford Hill antenna of the Bell Telephone Laboratories has also employed the Cassegrain configuration [27 - 29].

The feed of a Cassegrain or Gregorian antenna can either be mounted along the axis and near the vertex of the main reflector (i.e. an on-axis configuration) or tilted away from the axis (i.e. the off-axis and offset configurations). The off-axis antenna configuration is somewhat similar to 
the on-axis configuration. The main difference between these two configurations is that the sub-reflector and feed horn of the off-axis configuration are tilted and the feed horn is positioned away from the axis of the primary reflector. On the other hand, the sub-reflector and feed horn of an offset antenna are placed at one side of the primary reflector. Unlike the on-axis and off-axis designs where the focal point of the main reflector is along its axis, the focal point of the offset antenna is located at the side of the main reflector. Figures 1 to 3 show the offset, on-axis and off-axis Cassegrain configurations while Figures 4 to 6 depict those for the Gregorian configurations. The design and analysis of these antenna configurations, such as the calculation and measurement of the taper and spill-over loss, as well as, overall aperture efficiencies, could be easily found in the literatures [11 - 29]. It is to be noted, however, that comparison on the performance among the on-axis, off-axis, and offset Cassegrain and Gregorian designs is, surprisingly, rare. Since it will be interesting to find out which configuration performs better at a particular frequency, we present an investigation on the radiation characteristics of these reflector antennas in this paper.

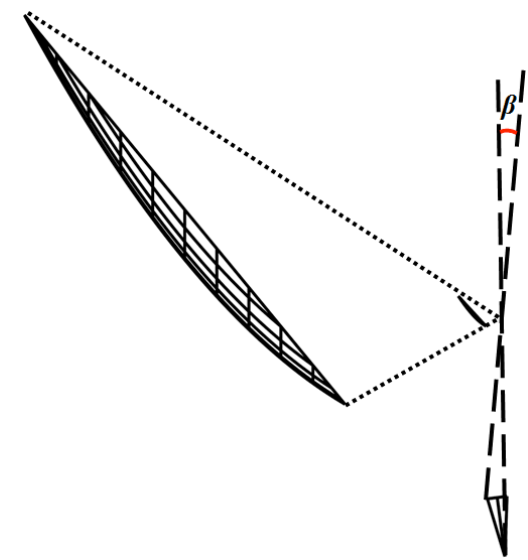

Figure 1: Optical arrangement of an offset Cassegrain antenna.

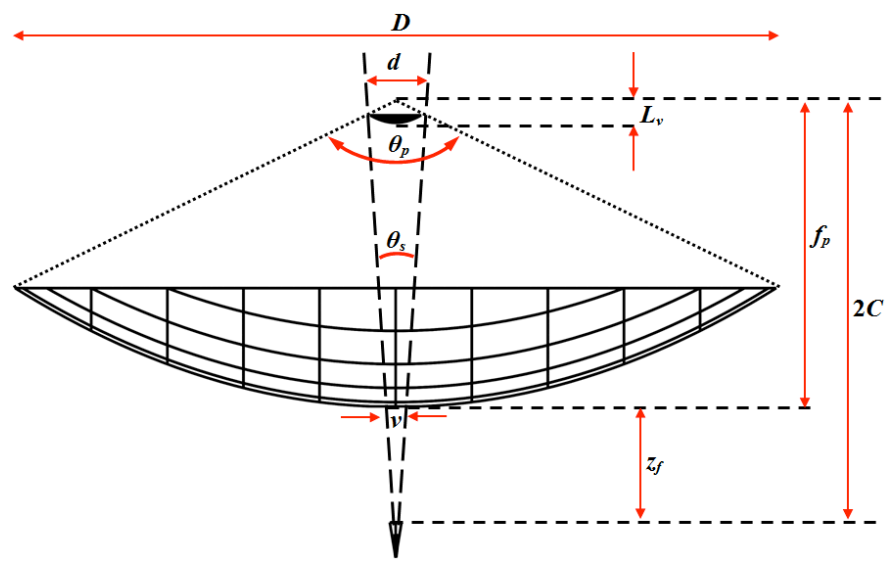

Figure 2: Optical arrangement of an on-axis Cassegrain antenna.

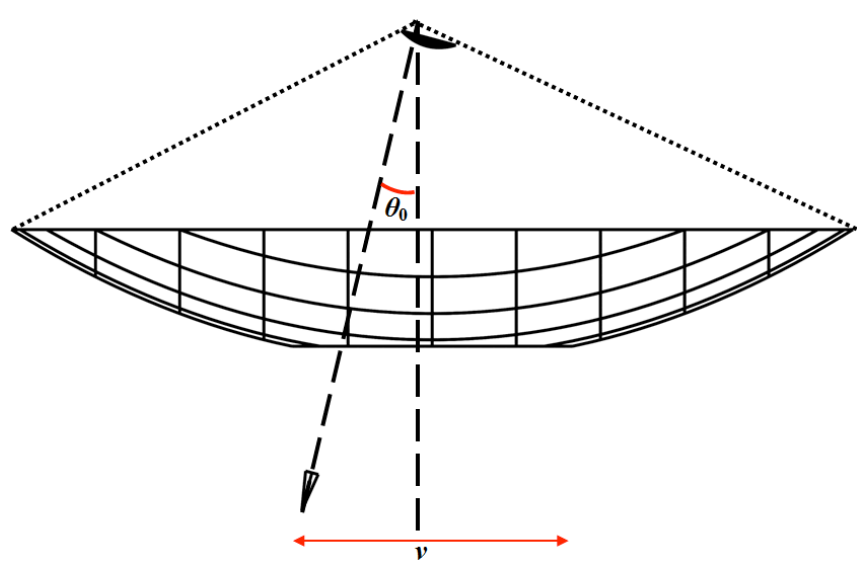

Figure 3: Optical arrangement of an off-axis Cassegrain antenna.

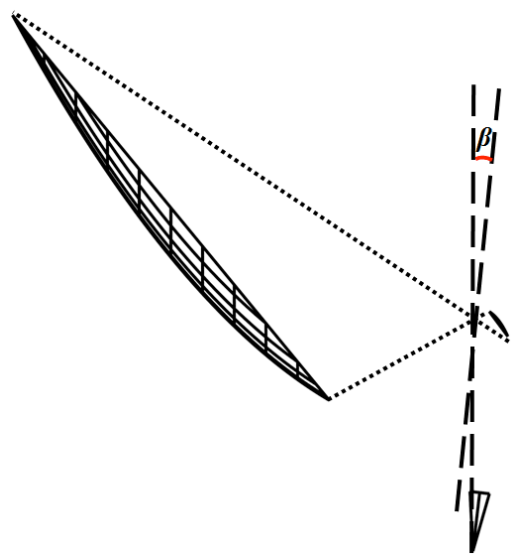

Figure 4: Optical arrangement of an offset Gregorian antenna.

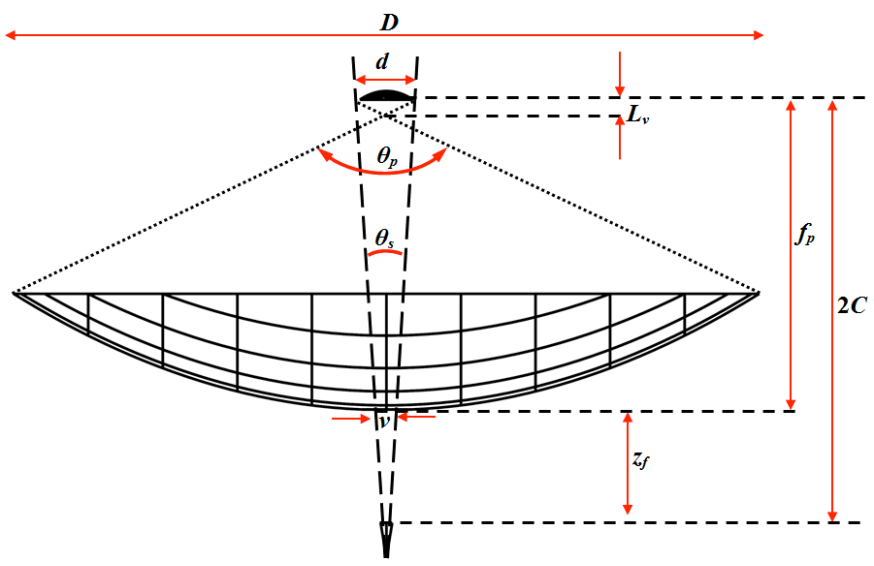

Figure 5: Optical arrangement of an on-axis Gregorian antenna. 


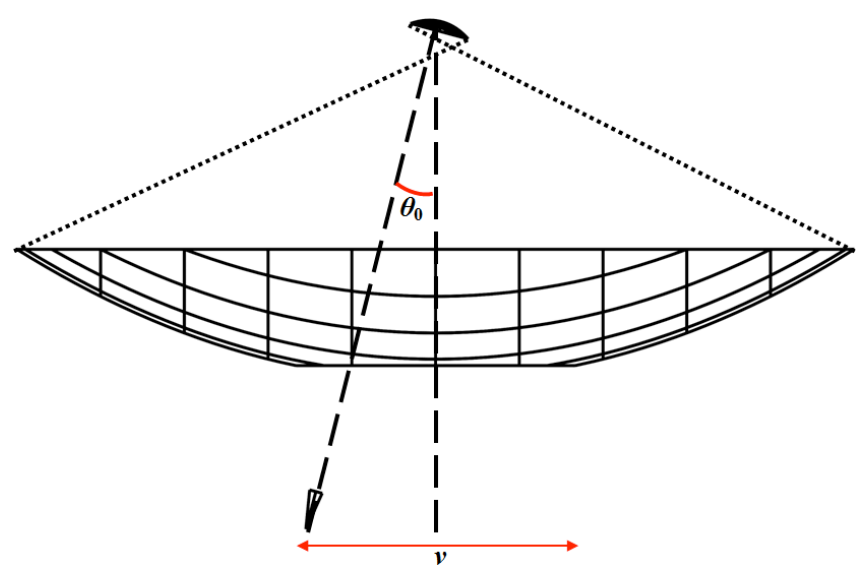

Figure 6: Optical arrangement of an off-axis Gregorian antenna.

\section{Design}

In our analysis, we employ the parameters used in [4] for both the on-axis Cassegrain and Gregorian antennas. These parameters are then modified to fit the design requirements of the offset and off-axis configurations. To minimize the side lobe levels and cross polarization, we apply Mizuguchi condition to optimize the design of our offset antennas. The condition to cancel cross polarization in offset designs can be determined by (1) below [30]

$$
M=\frac{\left|1-e^{2}\right|}{\left(1+e^{2}\right)-2 e \cos \beta}
$$

where $M$ is the magnification ratio, $e$ the eccentricity of the sub-reflector, and $\beta$ is the angle subtended between the axis of the primary reflector and that of the sub-reflector (as shown in Figures 1 and 4). To develop the offset configurations based on the original on-axis designs in [4], $\beta$ is adjusted to move the positions of the feed horn and the reflectors.

\section{Results and Discussion}

As shown in [31], the antenna design for ALMA band 1 gives the highest aperture efficiency at the high-edge frequency of $45 \mathrm{GHz}$. Hence, we have selected signal frequency $f$ at the high edge when analyzing the performance of the three types of designs, namely, on-axis, off-axis and offset for both Cassegrain and Gregorian configurations. For the offset designs, we have selected $\beta$ at $0.20^{\circ}$ based on the findings presented in [31]. In both Cassegrain and Gregorian offset designs, the smaller the angle of $\beta$, the distance of the feed horn will be shifted closer to the primary reflector. The feed horn is to be placed close to the reflector to minimize spillover loss. At the same time, care is also to be taken to make sure that the signal to be coupled to the feed horn would not be blocked by the reflector. As shown in [32], at $\beta=0.20^{\circ}$, the feed horn is placed close enough right next to the edge of the parabolic reflector, allowing it to receive the highest power. Tables 1 to 6 summarize the parameters for the design of the antennas. It is to be noted that the parameters for both the on-axis and off-axis designs are similar, with the exception that a much larger hole radius $v$ and an offset angle $\theta_{0}$ exist in the off-axis configurations.

In order to analyze the performance of the antennas, GRASP physical optics software has been used to simulate the radiation characteristics of the antennas. GRASP or General Reflector Antenna Software Package has been widely used in analyzing reflector antennas and antenna farms, particularly in the space industry. Indeed, the tool has been continuously refined based on industry requirements and backed by the European Space Agency (ESA). Experimental measurements for the ALMA receivers have been found to be in good agreement with those obtained from GRASP [33 - 36]. Figure 7 depicts the radiation patterns of the on-axis, off-axis and offset Cassegrain antennas. From the figure, it could be seen that the amplitude of the on-axis and the $0.20^{\circ}$ offset Cassegrain are comparable at the main lobe. The main lobe of the off-axis Cassegrain configuration, on the other hand, is much lower in amplitude; while the amplitude of its side lobes are relatively higher than those obtained from the other two counterparts. It is apparent that the off-axis design exhibits the lowest performance among all three designs. The poor performance of the off-axis Cassegrain can be attributed to the large hole radius $v$ at the primary reflector, which results in high spillover loss. The spillover efficiency $\varepsilon_{s}$ obtained from GRASP indicates that the offset Cassegrain is the highest, with $\varepsilon_{s}=0.9284$. This is followed by the on-axis with $\varepsilon_{s}=0.9169$ and lastly, the off-axis with $\varepsilon_{s}=0.6411$.

Figure 8 shows the radiation patterns of the on-axis, offaxis, and offset Gregorian antennas. Upon close inspection on both Figures 7 and 8, we could observe that the radiation patterns found in the Gregorian designs are similar with those in the Cassegrain designs. Like the case of the Cassegrain configurations, the off-axis design exhibits the lowest performance among all three Gregorian configurations - it has the lowest main lobe and high sidelobes. The spillover efficiency $\varepsilon_{s}$ of the offset, on-axis, and off-axis Gregorian designs are 0.9278, 0.9169, and 0.7840 , respectively.

It is to be noted that high spillover efficiency $\varepsilon_{s}$ does not necessarily guarantee high aperture efficiency $\varepsilon_{a}$ of an antenna. This is because the aperture efficiency $\varepsilon_{a}$ is governed by both spillover efficiency $\varepsilon_{s}$ and taper efficiency $\varepsilon_{t}$ [37]. The former corresponds to the degree of central concentration of an illumination distribution; whereas the latter corresponds to the degree of uniform illumination intercepted by the reflector. Since the most important indicator of an antenna design is the aperture efficiency $\varepsilon_{a}$, it is therefore computed here in order to determine the performances of the different kinds of antenna configurations. The aperture efficiency $\varepsilon_{a}$ can be expressed in terms of $\varepsilon_{s}$ as 


$$
\varepsilon_{a}=\frac{-4\left\{\exp \left[0.5\left(\frac{r_{s}}{r_{a}}\right)^{2} \ln \left(1-\varepsilon_{s}\right)\right]-\exp \left[0.5 \ln \left(1-\varepsilon_{s}\right)\right]\right\}^{2}}{\ln \left(1-\varepsilon_{s}\right)}
$$

where $r_{a}$ and $r_{s}$ denote the primary and secondary reflector radius, respectively. For the ALMA antenna, $r_{a}=6000 \mathrm{~mm}$ and $r_{s}=375 \mathrm{~mm}$. Table 7 summarizes the aperture efficiency $\varepsilon_{a}$ of the antennas. From the table, it can be seen that the on-axis design for both Cassegrain and Gregorian configurations are comparable with each other and that they exhibit the highest aperture efficiency $\varepsilon_{a}$. Although the offset configurations show the highest spillover efficiencies, their aperture efficiencies turn out to be lower than their on-axis counterparts. This result can be attributed to the lower taper efficiencies $\varepsilon_{t}$ of the configurations. The off-axis configurations show the worst performance among the designs. This is to be expected since the large centre hole radius $v$ at the primary reflector results in significant signal loss.

Table 1: Parameters for the offset Cassegrain antenna.

\begin{tabular}{|c|c|c|}
\hline Symbol & Description & Data \\
\hline$D$ & Diameter of primary aperture & $12.0 \mathrm{~m}$ \\
\hline$f_{p}$ & Focal length of primary reflector & $4.8 \mathrm{~m}$ \\
\hline$d$ & Diameter of secondary aperture & $0.75 \mathrm{~m}$ \\
\hline$M$ & Magnification & 20 \\
\hline$f / D$ & Primary focal ratio & 0.4 \\
\hline$F$ & $\begin{array}{c}\text { Focal length of equivalent } \\
\text { paraboloid }\end{array}$ & $96 \mathrm{~m}$ \\
\hline$F / D$ & Secondary focal ratio & 8 \\
\hline$e$ & Secondary eccentricity & 1.10526 \\
\hline $2 c$ & $\begin{array}{c}\text { Distance between primary and } \\
\text { secondary foci }\end{array}$ & $6.177 \mathrm{~m}$ \\
\hline$z_{f}$ & Back focal distance & $1.377 \mathrm{~m}$ \\
\hline$\beta$ & $\begin{array}{c}\text { Angle between main reflector axis } \\
\text { and secondary reflector axis }\end{array}$ & $0.20^{\circ}$ \\
\hline
\end{tabular}

Table 2: Parameters for the on-axis Cassegrain antenna.

\begin{tabular}{|c|c|c|}
\hline Symbol & Description & Data \\
\hline$D$ & Diameter of primary aperture & $12.0 \mathrm{~m}$ \\
\hline$f_{p}$ & Focal length of primary reflector & $4.8 \mathrm{~m}$ \\
\hline$d$ & Diameter of secondary aperture & $0.75 \mathrm{~m}$ \\
\hline$M$ & Magnification & 20 \\
\hline$f / D$ & Primary focal ratio & 0.4 \\
\hline$F$ & $\begin{array}{c}\text { Focal length of equivalent } \\
\text { paraboloid }\end{array}$ & $96 \mathrm{~m}$ \\
\hline$F / D$ & Secondary focal ratio & 8 \\
\hline$e$ & Secondary eccentricity & 1.10526 \\
\hline $2 c$ & $\begin{array}{c}\text { Distance between primary and } \\
\text { secondary foci }\end{array}$ & $6.177 \mathrm{~m}$ \\
\hline$z_{f}$ & Back focal distance & $1.377 \mathrm{~m}$ \\
\hline$\theta_{p}$ & Primary angle of illumination & $128.02^{\circ}$ \\
\hline$\theta_{s}$ & Secondary angle of illumination & $7.16^{\circ}$ \\
\hline$v$ & Primary vertex hole clear aperture & $0.75 \mathrm{~m}$ \\
\hline$L_{v}$ & $\begin{array}{c}\text { Distance between secondary vertex } \\
\text { and primary foci }\end{array}$ & $0.5886 \mathrm{~m}$ \\
\hline
\end{tabular}

Table 3: Parameters for the off-axis Cassegrain antenna.

\begin{tabular}{|c|c|c|}
\hline Symbol & Description & Data \\
\hline$D$ & Diameter of primary aperture & $12.0 \mathrm{~m}$ \\
\hline$f_{p}$ & Focal length of primary reflector & $4.8 \mathrm{~m}$ \\
\hline$d$ & Diameter of secondary aperture & $0.75 \mathrm{~m}$ \\
\hline$M$ & Magnification & 20 \\
\hline$f / D$ & Primary focal ratio & 0.4 \\
\hline$F$ & $\begin{array}{c}\text { Focal length of equivalent } \\
\text { paraboloid }\end{array}$ & $96 \mathrm{~m}$ \\
\hline$F / D$ & Secondary focal ratio & 8 \\
\hline$e$ & Secondary eccentricity & 1.10526 \\
\hline $2 c$ & $\begin{array}{c}\text { Distance between primary and } \\
\text { secondary foci }\end{array}$ & $6.177 \mathrm{~m}$ \\
\hline$z_{f}$ & Back focal distance & $1.377 \mathrm{~m}$ \\
\hline$\theta_{p}$ & Primary angle of illumination & $128.02^{\circ}$ \\
\hline$\theta_{s}$ & Secondary angle of illumination & $7.16^{\circ}$ \\
\hline$v$ & Primary vertex hole clear aperture & $4 \mathrm{~m}$ \\
\hline$\theta_{o}$ & Offset angle & $15^{\circ}$ \\
\hline
\end{tabular}

Table 4: Parameters for the offset Gregorian antenna.

\begin{tabular}{|c|c|c|}
\hline Symbol & Description & Data \\
\hline$D$ & Diameter of primary aperture & $12.0 \mathrm{~m}$ \\
\hline$f_{p}$ & Focal length of primary reflector & $4.8 \mathrm{~m}$ \\
\hline$d$ & Diameter of secondary aperture & $0.75 \mathrm{~m}$ \\
\hline$M$ & Magnification & -20 \\
\hline$f / D$ & Primary focal ratio & 0.4 \\
\hline$F$ & $\begin{array}{c}\text { Focal length of equivalent } \\
\text { paraboloid }\end{array}$ & $96 \mathrm{~m}$ \\
\hline$F / D$ & Secondary focal ratio & 8 \\
\hline$e$ & Secondary eccentricity & 0.90476 \\
\hline $2 c$ & $\begin{array}{c}\text { Distance between primary and } \\
\text { secondary foci }\end{array}$ & $6.177 \mathrm{~m}$ \\
\hline$z_{f}$ & Back focal distance & $1.377 \mathrm{~m}$ \\
\hline$\beta$ & $\begin{array}{c}\text { Angle between main reflector axis } \\
\text { and secondary reflector axis }\end{array}$ & $0.20^{\circ}$ \\
\hline
\end{tabular}

Table 5: Parameters for the on-axis Gregorian antenna.

\begin{tabular}{|c|c|c|}
\hline Symbol & Description & Data \\
\hline$D$ & Diameter of primary aperture & $12.0 \mathrm{~m}$ \\
\hline$f_{p}$ & Focal length of primary reflector & $4.8 \mathrm{~m}$ \\
\hline$d$ & Diameter of secondary aperture & $0.80 \mathrm{~m}$ \\
\hline$M$ & Magnification & -20 \\
\hline$f / D$ & Primary focal ratio & 0.4 \\
\hline$F$ & $\begin{array}{c}\text { Focal length of equivalent } \\
\text { paraboloid }\end{array}$ & $96 \mathrm{~m}$ \\
\hline$F / D$ & Secondary focal ratio & 8 \\
\hline$e$ & Secondary eccentricity & 0.90476 \\
\hline $2 c$ & $\begin{array}{c}\text { Distance between primary and } \\
\text { secondary foci }\end{array}$ & $6.177 \mathrm{~m}$ \\
\hline$z_{f}$ & Back focal distance & $1.377 \mathrm{~m}$ \\
\hline$\theta_{p}$ & Primary angle of illumination & $-128.02^{\circ}$ \\
\hline$\theta_{s}$ & Secondary angle of illumination & $7.16^{\circ}$ \\
\hline$v$ & Primary vertex hole clear aperture & $0.75 \mathrm{~m}$ \\
\hline$L_{v}$ & $\begin{array}{c}\text { Distance between secondary vertex } \\
\text { and primary foci }\end{array}$ & $0.6512 \mathrm{~m}$ \\
\hline
\end{tabular}


Table 6: Parameters for the off-axis Gregorian antenna.

\begin{tabular}{|c|c|c|}
\hline Symbol & Description & Data \\
\hline$D$ & Diameter of primary aperture & $12.0 \mathrm{~m}$ \\
\hline$f_{p}$ & Focal length of primary reflector & $4.8 \mathrm{~m}$ \\
\hline$d$ & Diameter of secondary aperture & $0.80 \mathrm{~m}$ \\
\hline$M$ & Magnification & -20 \\
\hline$f / D$ & Primary focal ratio & 0.4 \\
\hline$F$ & $\begin{array}{c}\text { Focal length of equivalent } \\
\text { paraboloid }\end{array}$ & $96 \mathrm{~m}$ \\
\hline$F / D$ & Secondary focal ratio & 8 \\
\hline$e$ & Secondary eccentricity & 0.90476 \\
\hline $2 c$ & $\begin{array}{c}\text { Distance between primary and } \\
\text { secondary foci }\end{array}$ & $6.177 \mathrm{~m}$ \\
\hline$z_{f}$ & Back focal distance & $1.377 \mathrm{~m}$ \\
\hline$\theta_{p}$ & Primary angle of illumination & $-128.02^{\circ}$ \\
\hline$\theta_{s}$ & Secondary angle of illumination & $7.16^{\circ}$ \\
\hline$v$ & Primary vertex hole clear aperture & $4 \mathrm{~m}$ \\
\hline$\theta_{o}$ & Offset angle & $15^{\circ}$ \\
\hline
\end{tabular}

Table 7: Aperture efficiencies of Cassegrain and Gregorian antennas.

\begin{tabular}{|l|c|c|}
\hline & Cassegrain & Gregorian \\
\hline Offset & $80.24 \%$ & $80.26 \%$ \\
\hline On-axis & $80.34 \%$ & $80.34 \%$ \\
\hline Off-axis & $62.11 \%$ & $73.94 \%$ \\
\hline
\end{tabular}

\section{Conclusions}

We have performed a comparison among on-axis, off-axis and offset designs for both Cassegrain and Gregorian reflectors. To reduce spillover loss in the offset design, we have adjusted the angle between the axis of the primary reflector and that of the sub-reflector to $0.20^{\circ}$ as presented in [31]. From the results, it is observed that the radiation patterns generated by the on-axis design are more superior compared to the offset and off-axis designs for both Cassegrain and Gregorian configurations. The radiation patterns from the on-axis designs for both antenna configurations are in close proximity between each other. The aperture efficiencies found in both on-axis Cassegrain and Gregorian designs are comparable with each other as well. Hence, it can be concluded that both on-axis Cassegrain and Gregorian antennas give the best performance.

\section{Acknowledgements}

Part of this work has been supported by the Fundamental Research Grant Scheme FRGS funded by the Ministry of Education, Malaysia (project: FRGS/2/2013/SG02/UTAR/02/1).

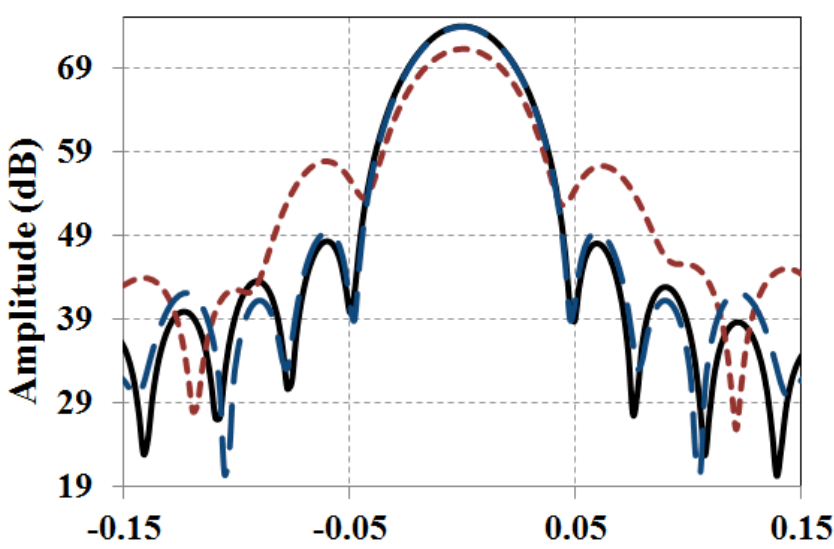

Angle (Degree)

(a)

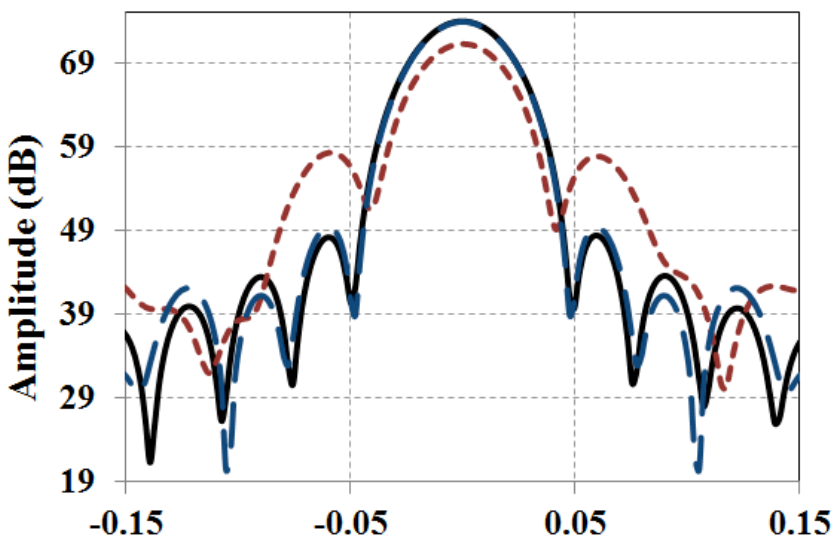

Angle (Degree)

(b)

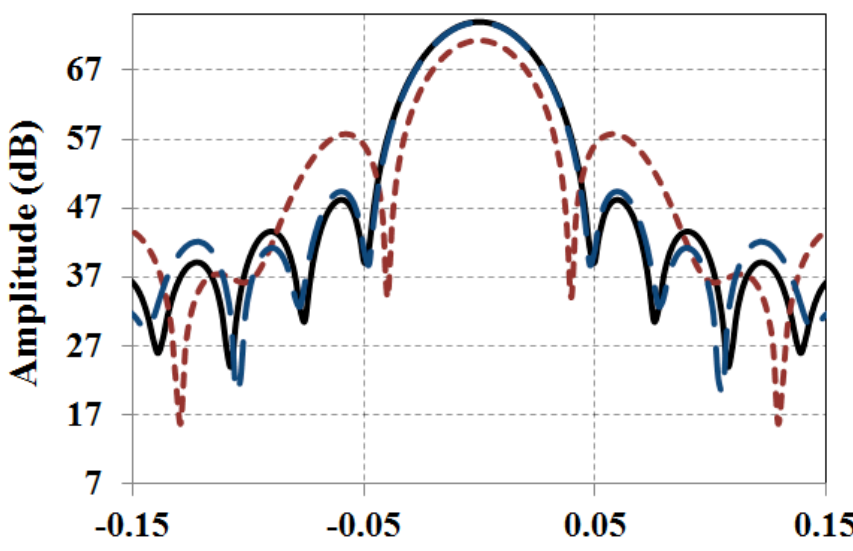

Angle (Degree)

(c)

Figure 7: The beam patterns of offset (solid lines), on-axis (dashed lines) and off-axis (dotted lines) Cassegrain antenna, at $f=45 \mathrm{GHz}$ and $\varphi=$ (a) $0^{\circ}$, (b) $45^{\circ}$ and (c) $90^{\circ}$. 


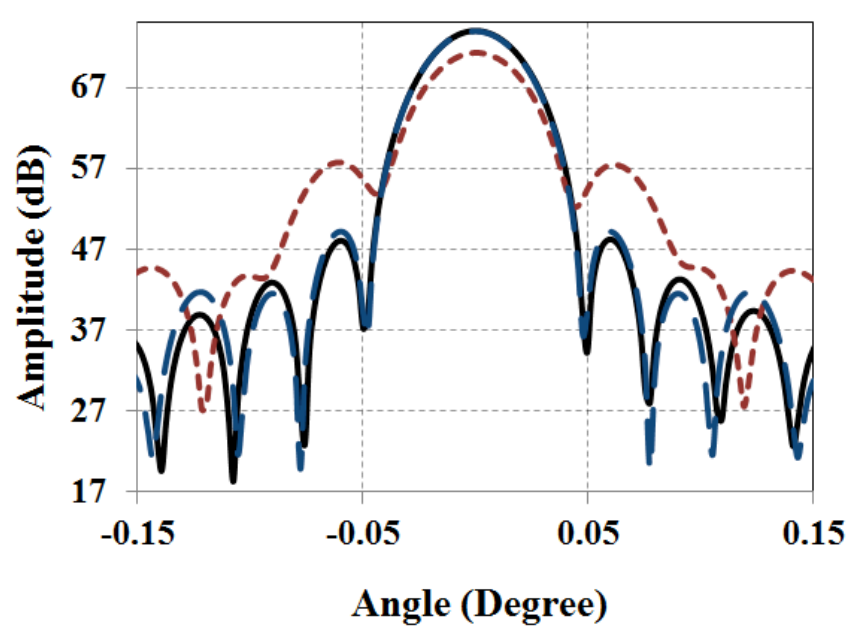

(a)

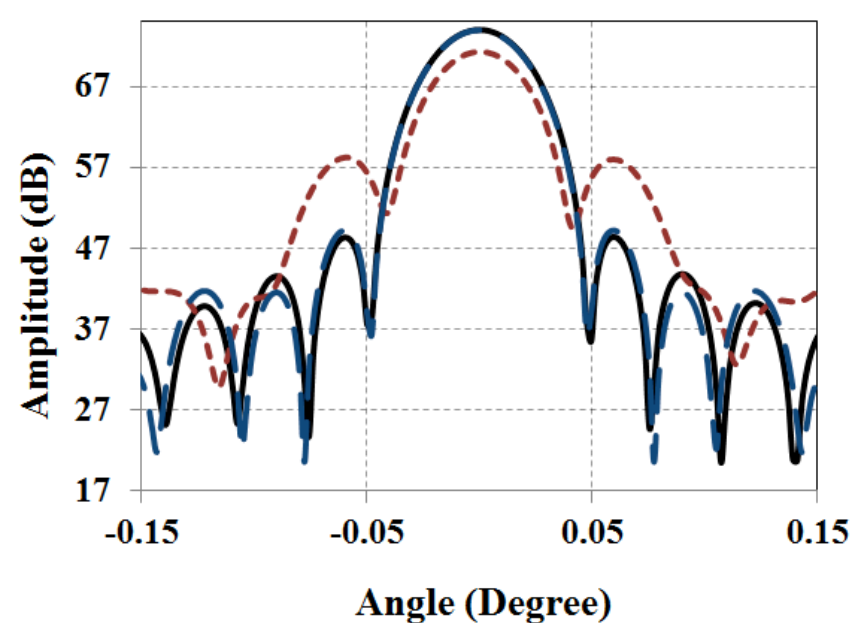

(b)

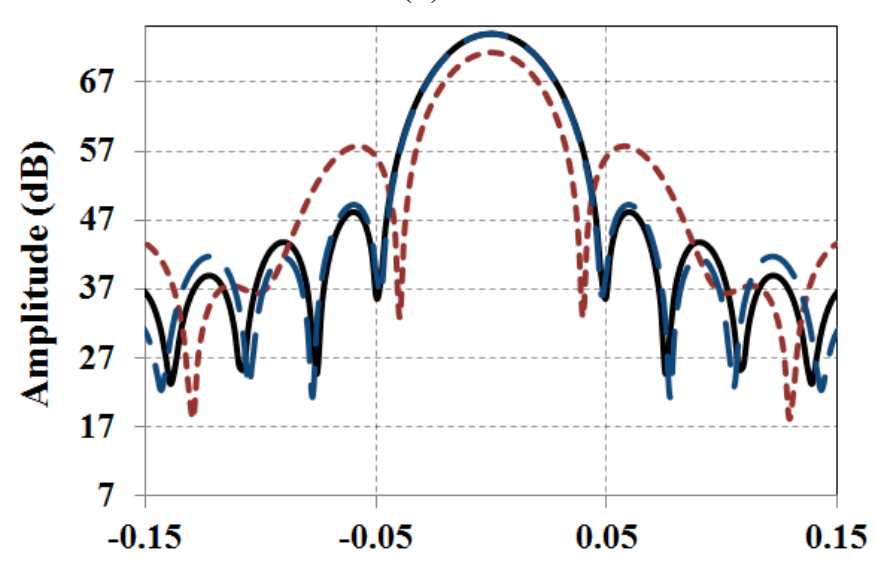

Angle (Degree)

(c)

Figure 8: The beam patterns of offset (solid lines), on-axis (dashed lines) and off-axis (dotted lines) Gregorian antenna, at $f=45 \mathrm{GHz}$ and $\varphi=$ (a) $0^{\circ}$, (b) $45^{\circ}$ and (c) $90^{\circ}$.

\section{References}

[1] T.G. Phllips, J. Keene, Submillimeter astronomy, Proc. of the IEEE, 80: 1662 - 1678, 1992.
[2] K.H. Yeap, C.Y. Tham, K.C. Yeong, K.H. Chong, Z.I. Rizman, C.C. Yang, Analysis of normal and superconducting coplanar waveguides in radio astronomy, Int. J. of Electronics, Computer, and Communication Technologies, 2: 9 - 12, 2011.

[3] J.C. Cheng, E.S. Cheng, D.A. Cottingham, R.E. Eplee, D.J. Jr. Fixsen, T. Hewagama, R.B. Isaacman, K.A. Jensen, S.S. Meyer, P.D. Noerdlinger, S.M. Read, L.P. Rosen, R.A. Shafer, E.L. Wright, C.L. Bennett, N.W. Boggess, M.G. Hauser, T. Kelsall, S.H. Moseley, R.F. Jr. Silverberg, G.F. Smoot, R. Weiss, D.T. Wilkinson, Measurement of the cosmic microwave background spectrum by the COBE FIRAS instrument, The Astrophysical J, 410: 439 - 444, 1994.

[4] K.H. Yeap, Y.H. Law, Z.I. Rizman, Y.K. Cheong, C.E. Ong, K.H. Chong, Performance analysis of paraboloidal reflector antennas in radio telescopes, Int. J. of Electronics, Computer, and Communication Technologies, 4: 21 - 25, 2013.

[5] A. Wotten, ALMA capabilities for observations of spectral line emission, Astrophysics and Space Science, 313: $9-12$, 2008.

[6] K.H. Yeap, C.Y. Tham, H. Nisar, S.H. Loh, Analysis of probes in a rectangular waveguide, Frequenz $J$. of $R F$ Engineering and Telecommunications, 67: 145 - 154, 2013.

[7] K.H. Yeap, C.Y. Tham, G. Yassin, K.C. Yeong, Attenuation in rectangular waveguides with finite conductivity walls, Radioengineering J., 20: 472 - 478, 2011.

[8] S. Paine, D.C. Papa, R.L. Leombruno, X. Zhang, R. Blundell, Beam waveguide and receiver optics for the SMA, Proc. of the 5th Int. Symp. on Space THz Technology, University of Michigan, Ann Arbor, Michigan, 1994.

[9] C.A. Balanis, Antenna Theory: Analysis and Design, Wiley Interscience, $3^{\text {rd }}$ ed., pp. 883 - 934, 2005.

[10] T.L. Wilson, K. Rohlfs, S. Huttemeister, Tools of Radio Astronomy: Astronomy and Astrophysics Library, Springer, 5th ed., pp. $154-157,2009$.

[11] J.L. Han, P.B. Demorest, W.V. Straten, A.G. Lyne, Polarization observations of 100 pulsars at $774 \mathrm{MHz}$ by the Green Bank Telescope, The Astrophysical Journal Supplement Series, 181: 557 - 571, 2009.

[12] S.M. Ransom, P.S. Ray, F. Camilo, M.S.E. Roberts, O. Celik, M.T. Wolff, C.C. Cheung, M. Kerr, T. Penucci, M.E. Decesar, I. Cognard, A.G. Lyne, B.W. Stappers, P.C.C. Freire, J.E. Grove, A.A. Abdo, G. Desvignes, D. Donato, E.C. Ferrara, N. Gehrels, L. Guillemot, C. Gwon, A.K. Harding, S. Johnston, M. Keith, M. Kramer, P.F. Michelson, D. Parent, P.M.S. Parkinson, R.W. Romani, D.A. Smith, G. Theureau, D.J. Thompson, P. Weltevrede, K.S. Wood, M. Ziegler, Three millisecond pulsars in Fermi Lat unassociated bright sources, The Astrophysical Journal Letters, 727: 1 - 6, 2011.

[13] T.R. Hunter, F.R. Schwab, S.D. White, J.M. Ford, F.D. Ghigo, R.J. Maddalena, B.S. Mason, J.D. Nelson, R.M. Prestage, J. Ray, P. Ries, R. Simon, S. Srikanth, P. Whiteis, Holographic measurement and improvement of the Green Bank Telescope surface, Publications of the Astronomical Society of the Pacific, 123: 1087 - 1099, 2011.

[14] R.M. Prestage, K.T. Constantikes, T.R. Hunter, L.J. King, R.J. Lacasse, F.J. Lockman, R.D. Norrod, The Green Bank Telescope, Proc. of the IEEE, 97: 1382 - 1290, 2009.

[15] I. Seker, J.D. Mathews, J. Wiig, P.F. Gutierrez, J.S. Friedman, C.A. Tepley, First results from the Penn state allsky imager at the Arecibo observatory, Earth Planets Space, 59: 165 - 176, 2007. 
[16] J.D. Mathews, J. Doherty, C.-H.Wen, S.J. Briczinski, D. Janches, D. D. Meisel, An update on UHF radar meteor observations and associated signal processing techniques at Arecibo observatory, J. of Atmospheric and Solar-Terrestrial Physics, 65: 1139 - 1149, 2003.

[17] A.P.V. Siemion, G.C. Bower, G. Foster, P.L. McMahon, M.I. Wagner, D. Werthimer, D. Backer, J. Cordes, J.V. Leeuwen, The Allen telescope array fly's eye survey for fast radio transients, The Astrophysical J., 744: 109, 2012.

[18] S. Croft, G.C. Bower, G. Keating, C. Law, D. Whysong, P.K.G. Williams, M. Wright, The Allen telescope array twenty-centimeter survey - A 700-square-degree, multi-epoch radio data set. II. Individual epoch transient statistics, The Astrophysical J., 731: 34, 2011.

[19] A. Young, M.A.B. Terada, D.I.L. de Villiers, D. B. Davidson, Assessment of the sensitivity of the south African KAT-7 and MEERKAT/SKA radio telescope reflector antennas, Proc. of the Int. Conf. on Electromagnetics in Advanced Applications, Cape Town, pp. 486 - 489, 2012.

[20] D.I.L. de Villiers, Offset dual-reflector antenna system efficiency predictions including subreflector diffraction, IEEE Antennas and Wireless Propagation Letters, 10: 947 - 950, 2011.

[21] D.I.L. de Villiers. Prediction of aperture efficiency ripple in clear aperture offset Gregorian antennas, IEEE Trans. on Antennas and Propagation, 61: 2457 - 2465, 2013.

[22] G. Cortes-Medellin, Non-planar quasi-self-complementary ultra-wideband feed antenna, IEEE Trans. on Antennas and Propagation, 59: 1935 - 1944, 2011.

[23] A. Gonzalez, Y. Uzawa, Y. Fujii, K. Kaneko, ALMA band 10 tertiary optics, Infrared Physics and Technology, 54: $488-$ 496, 2011.

[24] C.Y. Tham, G. Yassin, M. Carter, Analysis techniques for the optics in millimeter/submillimeter wave radio telescope receivers, Jurnal Fizik Malaysia, 28: 49 - 53, 2007.

[25] M. Candotti, A.M. Baryshev, N. Trappe, Quasi-optical assessment of the ALMA band 9 front-end, Infrared Physics and Technology, 5: 174-179, 2007.

[26] C.Y. Tham, S. Withington, ALMA receiver optics design, electromagnetic analysis, second report (band 3, 6, 7, and 9), 2003.

[27] W. Rusch, The current state of the reflector antenna are entering the 1990s, Proc. of the IEEE, 80: 113 - 126, 1992.

[28] J.S. Courtney-Pratt, J.H. Hett, J.W. McLaughlin, Optical measurements on Telstar to determine the orientation of the spin axis, and the spin rate, J. Society of Motion Picture and Television Engineers, 72: 462 - 484, 1963.

[29] T.A. Milligan, Modern Antenna Design, Wiley Interscience. 2nd ed., 2005.

[30] Y. Mizugutch, M. Akagawa, H. Yokoi, Offset dual reflector antenna, Int. Symp. of Antennas and Propagation Society, 14: $2-5,1976$.

[31] J. W. Lamb, A. Baryshev, M. C. Carter, L. R. D’Addario, B. N. Ellison, W. Grammer, B. Lazareff, Y. Sekimoto, and C. Y. Tham. (2001, Apr.). ALMA Receiver Optics Design. ALMA Memo 362, The Atacama Large Millimetre Array Project, National Radio Astronomy Observatory.

[32] K.H. Yeap, C.Y. Yiam, Y.H. Ling, M.C. Loh, H. Nisar, K.C. Lai, Z.I. Rizman, Analysis of offset reflector antennas in radio telescopes, Proc. of the 2nd Int. Sc., Tech. and Eng. Conference, Penang, Malaysia, 2016.
[33] M. Candotti, A. Baryshev, N. Trappe, Quasi-optical assessment of the ALMA band 9 front-end, Infrared Physics and Technology, 52: 174-179, 2009.

[34] M. Candotti, A. Baryshev, N.A. Trappe, R. Hesper, J.A. Murphy, J. Barkhof,. Quasi-optical verification of the band 9 ALMA front-end, Proc. of the 16th Int. Symp. on Space THz Tech., Chalmers University of Technology, Goteborg, Sweden, pp. $79-84,2009$.

[35] M. Naruse, T. Ito, Y. Sekimoto, H. Toba, N. Satou, M. Sugimoto, W. Shan, Y. Iizuka, T. Kamba, M. Kamikura, Y. Serizawa, Near-field beam pattern measurement of qualification model of ALMA band $8(385-500 \mathrm{GHz})$ cartridge receiver, Exp. Astron., 24: 89 - 107, 2009.

[36] Y. Sekimoto, Y. Iizuka, N. Satou, T. Ito, K. Kumagai, M. Kamikura, M. Naruse, W.L. Shan, Development of ALMA band $8(385-500 \mathrm{GHz})$ Cartridge. Proc. of the 19th Int. Symp. on Space THz Tech., Groningen, Netherlands, pp. 253 257, 2008.

[37] P. F. Goldsmith, Quasioptical Systems; Gaussian Beam, Quasioptical Propagation and Applications, New York: IEEE Press, 1998. 